\title{
Spatial computation with gamma oscillations
}

\author{
Ben Engelhard ${ }^{1,2} *$ and Eilon Vaadia ${ }^{1,2}$ \\ 'Department of Medical Neurobiology, Institute of Medical Research Israel-Canada, The Hebrew University Hadassah Medical School, Jerusalem, Israel \\ ${ }^{2}$ Edmond and Lily Safra Center for Brain Sciences, The Interdisciplinary Center for Neural Computation, The Hebrew University of Jerusalem, Jerusalem, Israel
}

Edited by:

Zoltan Nadasdy, Seton Brain and

Spine Institute, USA

Reviewed by:

Ulrik R. Beierholm, University of Birmingham, UK

Hugo Merchant, Universidad

Nacional Autónoma de México,

Mexico

*Correspondence:

Ben Engelhard, Edmond and Lily Safra Center for Brain Sciences,

The Interdisciplinary Center for

Neural Computation, The Hebrew

University of Jerusalem, Silberman

building, 6th floor, Edmond J. Safra

Campus at Givat Ram, Jerusalem

91904, Israel

e-mail:ben.engelhard@

mail.huji.ac.il
Gamma oscillations in cortex have been extensively studied with relation to behavior in both humans and animal models; however, their computational role in the processing of behaviorally relevant signals is still not clear. One oft-overlooked characteristic of gamma oscillations is their spatial distribution over the cortical space and the computational consequences of such an organization. Here, we advance the proposal that the spatial organization of gamma oscillations is of major importance for their function. The interaction of specific spatial distributions of oscillations with the functional topography of cortex enables select amplification of neuronal signals, which supports perceptual and cognitive processing.

Keywords: gamma oscillations, functional topography, temporal synchrony, phase coding, cortical computation

\section{INTRODUCTION}

Neuronal oscillations reflect synchronized network activity important for brain computation (Buzsáki, 2006). Such oscillations have been recorded using electrodes placed on the scalp (Berger, 1929), intracranially on the brain surface (Jacobs and Kahana, 2010) and implanted in the brain itself, allowing for recording in both cortical and subcortical structures (Buzsáki and Draguhn, 2004; Schnitzler and Gross, 2005).

In this perspective we focus on oscillations in the gamma band, typically described in the range of $30-70 \mathrm{~Hz}$. Gamma activity has been related to many behavioral conditions including cognitive functions (Herrmann et al., 2010; Siegel et al., 2012). Additionally, clinical interest in this frequency band has increased following studies which have linked abnormal gamma activity with various brain disorders, including Schizophrenia, Autism, ADHD, Alzheimer's Disease and others (Uhlhaas and Singer, 2006, 2012; Bašar and Güntekin, 2008). Thus, the study of the function and mechanisms of gamma oscillations is significant for basic science as well as for clinical applications. Computationally, gamma-band activity has been hypothesized to reflect the temporally synchronized firing of single units, which could serve as a basis for associations of neuronal ensembles (Gray and Singer, 1989; Singer and Gray, 1995). However, the functional role of gamma activity and the computational means by which it is achieved is still unclear (Palanca and DeAngelis, 2005; Fries, 2009; Wang, 2010; Burns et al., 2011). Here, we propose that gamma activity reflects an oscillatory synchronization process distributed in a spatially-specific manner, which is characterized by precise-time correlation of single neurons that enables coincident firing onto target populations. The flexibility of the proposed computation lies in the interplay between the spatial distribution of the oscillatory process, the amplitude of oscillations, and the modulations of firing rates of tuned neuronal populations.

\section{PHASE-LOCKED FIRING AMPLIFIES EFFICACY OF CONVERGENT ACTIVITY}

Temporal synchrony in spike firing results in an increase in coincident events (Abeles, 1991; König et al., 1996). What would the effect of such synchrony be on target neuronal populations? Evidence suggests that cortical neurons have enhanced sensitivity to synchronous inputs (Abeles, 1982; Azouz and Gray, 2000, 2003; London and Häusser, 2005). Accordingly, by coincidence detection, synchronous signals would be amplified (König et al., 1996). In fact, this phenomenon has been reported in thalamocortical circuits, where weak thalamic projections to cortex induce strong feed-forward excitation by way of synchronous activity of convergent inputs (Bruno and Sakmann, 2006). Thus, temporal synchrony can amplify the effect of neuronal activity without requiring an increase in the firing rate of the inputs. How is this synchrony achieved in the presence of oscillations? There is evidence that when cortical networks enter a state characterized by gamma oscillations, neurons fire in a phase-locked manner, hence increasing the temporal synchrony between them (Singer and Gray, 1995). Further, we have recently shown that the relationship between the strength of gamma oscillations in the local field potentials (LFP) and the strength of precise-time spike synchrony is quantitative (Engelhard et al., 2013; see Figure 1); namely, periods with increased amplitude of gamma oscillations are characterized by stronger pairwise interactions as well as by 
an increase in the size of precisely synchronized assemblies. Thus, during a strong oscillatory state of the network (in the gamma band) the increase in temporal synchrony would amplify the signals produced by neurons in the oscillating region as long as their output converges onto target populations.

\section{OSCILLATION STRENGTH AND PRECISE-TIME SYNCHRONY}

Consider two cortical sites where there is need for temporal synchronization of spike-firing in both sites. What conditions are required for this synchronization by an oscillatory mechanism to take place? First, since the level of temporal synchronization depends on the strength of the oscillations, oscillations in both sites should increase simultaneously. Thus we would expect a correlation between the amplitude of field oscillations in both sites. Second, since the spikes are phase-locked to the oscillations, there needs to be phase coherence (or correlation) between the oscillations in both sites. We also expect the phase lag between oscillations to be small, (for nearby sites) or at least coordinated to optimize the required effects of the sites on the target neuronal population. Third, there should be a tight clustering of the preferred firing phases of neurons in both sites, so that the temporal coincidence of firing is maximal. The second and third conditions constrain the preferred firing time of neurons to be in tight coordination, which is important to reduce the detrimental effect of the temporal jittering noise of spiking around the preferred firing phase.

There is evidence for these three conditions in cortex. Amplitude correlations of gamma activity have been found in distributed sites during a visual comparison task (Bruns et al., 2000). Zero-lag coherent oscillations have been found in relatively nearby as well as distant sites (König et al., 1995). In our study, where monkeys were operantly trained to increase the power of gamma oscillations of the LFP in a subset of four electrodes out of a 96 electrode-array, we found both a strong amplitude and phase correlation between oscillations in the conditioned sites, and small phase differences between them (Engelhard et al., 2013; see Figure 1). The small scale of our array ( $400 \mu \mathrm{m}$ interelectrode distance) complements the larger scale findings and alludes to the pervasiveness of this phenomenon. Additionally, we found tight clustering of the preferred phases of firing, so that most neurons preferentially fired in the falling flank of LFP, as has been shown elsewhere (Murthy and Fetz, 1996; Denker et al., 2011).

The data reported in Hasenstaub et al. (2005) further supports this view. They showed that although fast spiking interneurons and regular spiking cells have a different mean overall preferred firing phase, both these populations have a relatively narrow range of preferred phases of firing in the gamma cycle. The standard deviation of the preferred firing phases for regular spiking cells was $2.4 \mathrm{~ms}$; such temporal clustering in the preferred firing phase across the population would enable coincident firing for neurons in sites characterized by high oscillation amplitude, as proposed here. Another study (Womelsdorf et al., 2012) showed similarly tight clustering in the primary visual cortex of awake monkeys.

It is important to note that the type of computation-bycoincidence described here does not require a perfectly regular unfolding gamma phase, as the amplification effect on target populations can be achieved whenever coincident firing occurs, regardless of the regularity between coincident episodes. This is significant because cortical gamma oscillations may exhibit variability in their phase distribution across cycles (Burns et al., 2011).

Finally, if the level of oscillations is involved in computations related to cognitive tasks, we would expect to observe a relationship between oscillation strength and behaviorally relevant parameters. Such relationships have been widely observed in different tasks and brain areas. For example, in the primary visual cortex, gamma power was shown to depend on the level of stimulus contrast (Logothetis et al., 2001; Henrie and Shapley, 2005). In the primary somatosensory cortex, gamma power changed in relation to different levels of subjective pain intensity (Zhang et al., 2012). In the posterior parietal cortex, there were changes in gamma power corresponding to the planning of different movement behaviors (Scherberger et al., 2005).

\section{SPATIAL DISTRIBUTION OF OSCILLATIONS}

In this section we first address the benefits of spatially-based computations in cortex; we then ask which experimental findings would be suggestive that gamma oscillations are involved in such computations, and proceed to review the presence of these findings in the literature.

Above, we discussed how precise-time synchrony can amplify the signal of the synchronized neurons, and showed that when the network enters an oscillatory state such synchrony can be achieved. However, in order to provide a substrate for effective computations this amplification should be specific to task-related neuronal assemblies. The spatial distribution of oscillations could be a mechanism to enable specific amplification output signals in particular cortical areas. The plausibility of this hypothesis stems from the finding that the spatial pattern of gamma oscillations is tightly linked to the spatial pattern of precise-time synchrony (Engelhard et al., 2013).

Functional topographic organization is a prominent feature in sensory cortex, and reflects the orderly convergence of afferents to adjacent sites (Mesulam, 1998). In the motor cortex, apart from the gross homunculus (Penfield and Boldrey, 1937), functional mapping is much less clear (Schieber and Hibbard, 1993; Ben-Shaul et al., 2003; Rathelot and Strick, 2006; but see Georgopoulos et al., 2007). In fact, the wide intermingling of cortico-muscular neurons in different muscle-related areas has led to the view that functional diversity across the cortical space is conducive to the planning and production of movements involving different muscle synergies (Rathelot and Strick, 2006). This view has also garnered support from electrical micro-stimulation studies showing that natural movements can be evoked by sitespecific stimulation (Graziano et al., 2002). Thus, in many cortical areas, focal activity of nearby neurons seems to be functionally relevant. A mechanism that exploits these relations by activating spatially-defined populations that are behaviorally congruent and with sufficiently rapid activation timescales would therefore be practicable for perception, motor functions and cognitive processing.

If gamma oscillations are related to spatial computations in cortex, we would expect experimental evidence for stimulus 
A

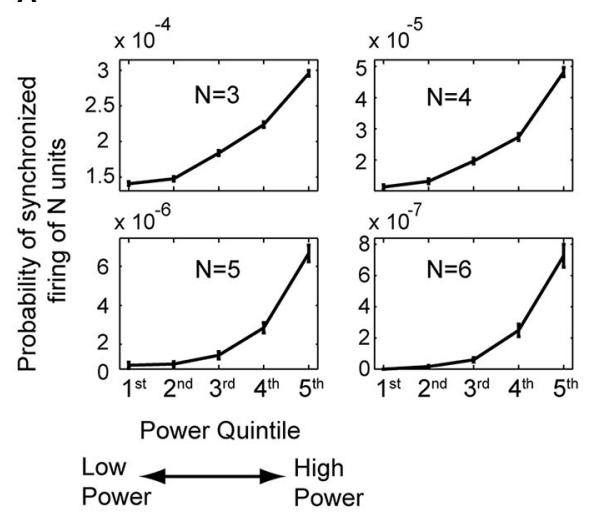

D

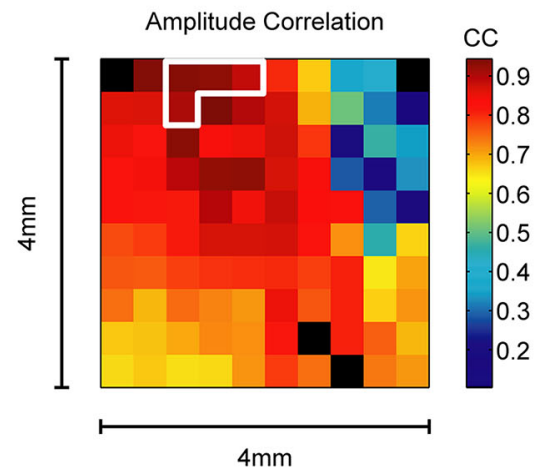

B

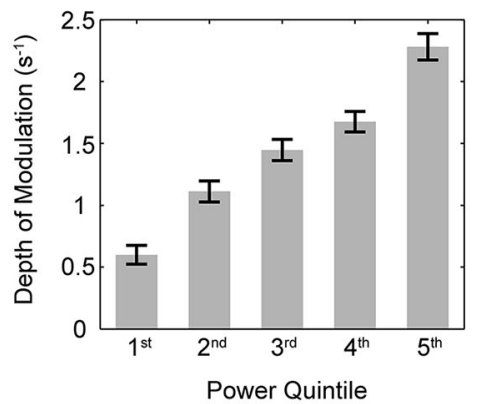

E

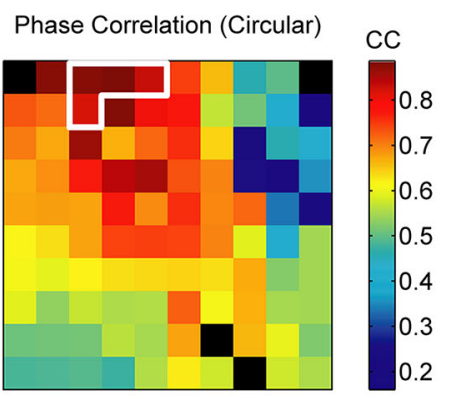

C

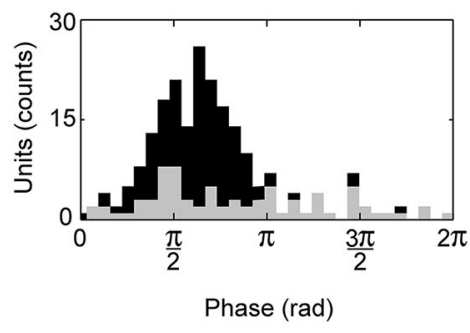

$\mathbf{F}$

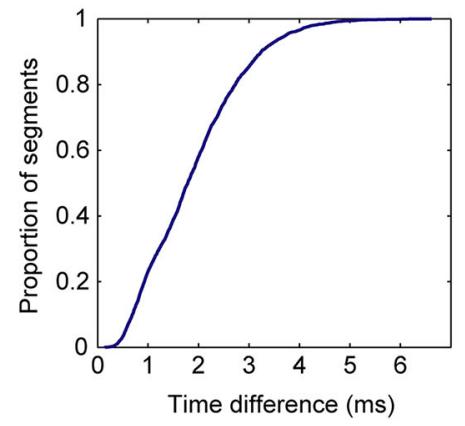

FIGURE 1 | Relationship between oscillation strength, single neuron synchrony, and oscillatory properties across the cortical space. (A) Probability of $\mathrm{N}$ recorded units to fire together in a $5 \mathrm{~ms}$ bin with $N$ shown in each frame, for $N=3$ to $N=6$. This probability was calculated separately for five periods of increasing power of low-gamma oscillations $(30-43 \mathrm{~Hz})$, shown on the $x$ axis. Error bars are SEM. The size of synchronized ensembles increased with the amplitude of oscillations. (B) Mean \pm SEM of the depth of modulation of the cross-correlation histograms for five periods of increasing power of oscillations $(30-43 \mathrm{~Hz})$, shown on the $x$ axis. Data are from pairs that exhibited significantly increased depth of modulation in segments of high oscillation power. The depth of modulation is a measure of the strength of pairwise neuronal interactions. (C) Histogram of the preferred phases of firing for all single units in segments of high oscillation power. Counts of significant preferred phases are marked in black (Raleigh test, $p<0.0001$ with Bonferroni correction). $63.6 \%$ of the 218 single units had a significant preferred firing phase. Note the tight temporal clustering of the preferred phases of firing. (D) Mean correlations of the instantaneous amplitude of $30-43 \mathrm{~Hz}$ oscillations of LFP between all sites and sites conditioned for an increase in power (circled in white). Data are from one recording day and for segments of high oscillation power. Note the spatial distribution of oscillations (which is not circular) and high correlations near the conditioned sites. The recording array was $4 \times 4 \mathrm{~mm}$ and had $400 \mu \mathrm{m}$ interelectrode spacing. Black squares are non-recording electrodes. (E) Mean circular correlations between the instantaneous $30-43 \mathrm{~Hz}$ phase in all sites and sites conditioned for an increase in power (circled in white). Data are the same as in (D). (F) Cumulative distribution of mean absolute time differences between phases of the oscillations in the conditioned sites for segments of high oscillation power. Data are the same as in (D). Note the small time differences for the majority of the segments. Panels (A-C) adapted from Engelhard et al. (2013). (or task) dependence of the spatial distribution of oscillations across the cortical space with oscillations that are stronger in areas functionally relevant to the task or stimulus. These phenomena have been reported in cortex. The stimulus dependence of the spatial distribution of oscillations was clearly evidenced in both V1 and V4 for visual stimuli (Rols et al., 2001; see Figure 2). Further, the topography of oscillations followed the retinotopic cortical map. Similarly, gamma activity related to attention was found to be localized in regions responsive to the particular stimulus in V4 (Taylor et al., 2005). Such stimulus- or taskspecific spatial distributions have also been found in a short-term memory task (Tallon-Baudry et al., 1998) and during presentations of coherent visual patterns (Lutzenberger et al., 1995).
An electrocorticography (ECoG) study found stimulus-specific spatial distributions of gamma activity in response to visually presented letters (Jacobs and Kahana, 2009). In the auditory cortex of monkeys, stimulus-dependent gamma oscillations have been described with spatial configurations that depend on the specific frequency response properties of the recorded sites (Brosch et al., 2002).

Further support for this idea comes from a study which showed that specific thalamic stimulation evokes gamma oscillations in modality-specific cortical areas (Macdonald et al., 1998). This demonstrates the presence of thalamocortical circuits capable of inducing specific spatial distributions of gamma oscillations. 
A
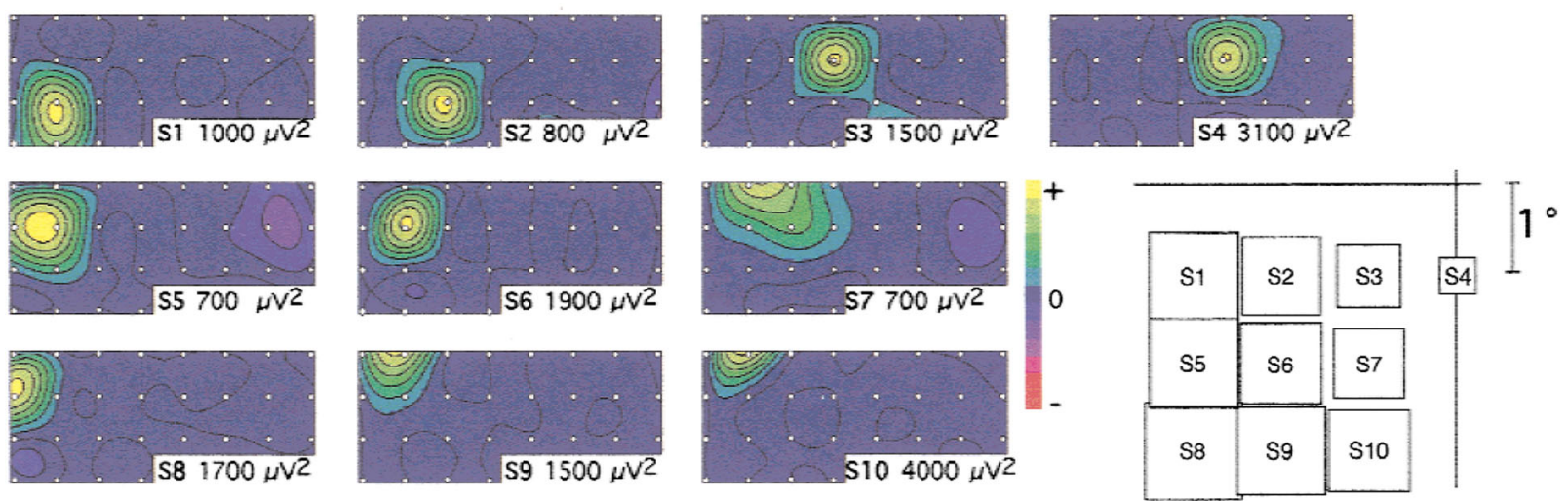

B
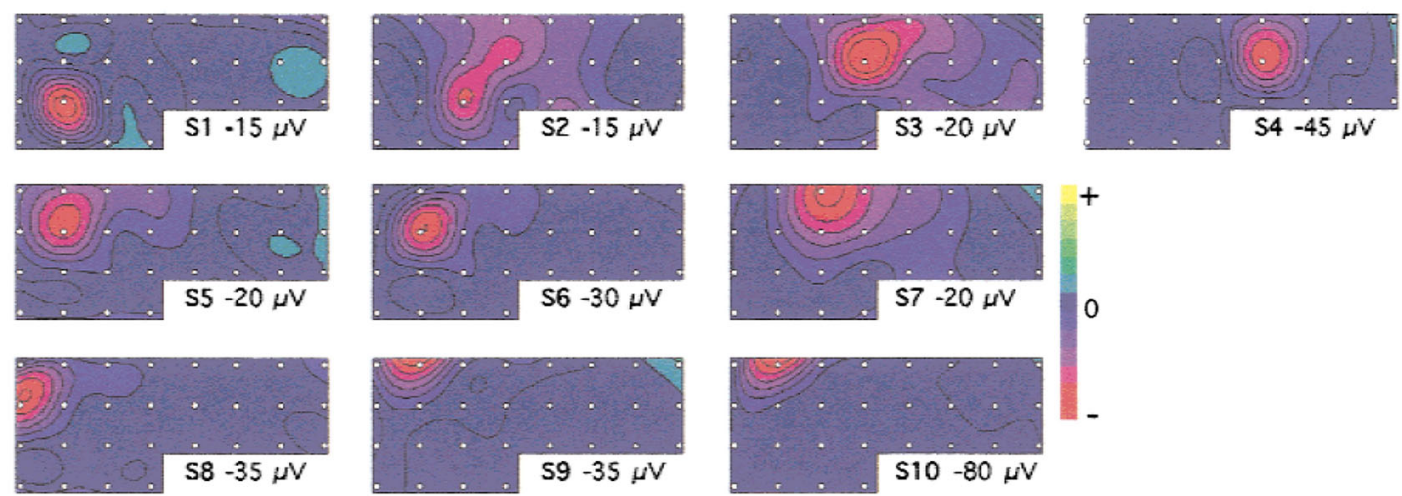

FIGURE 2 | Spatial distribution of gamma activity across the cortical space in V1. (A) Topographical distribution of the sustained gamma oscillations $(30-70 \mathrm{~Hz})$ at the different stimulus locations tested (indicated on the right). (B) Topographical distribution of the first negative component of the evoked potential, for the same stimuli, at $64 \mathrm{~ms}$. Note the stimulus-dependent spatial distribution of the gamma oscillations and evoked component. ECoG was recorded using an implanted array with $3 \mathrm{~mm}$ interelectrode spacing. Figure reproduced with permission from Rols et al. (2001).

\section{NEURONAL DIVERSITY AND TUNED POPULATIONS}

While in theory the described spatial computation would work well in a cartoon cortex with perfect topography, in actuality there is a wide diversity of function in cortex, even in areas that exhibit a clear functional topography (Ringach et al., 2002; Rothschild et al., 2010). This diversity is compounded in areas where such topography is much less clear, such as the primary motor cortex (Rathelot and Strick, 2006). Thus, there needs to be a mechanism to enable differentiation between nearby neurons with widely differing functions. We propose that neuronal tuning via modulation of their firing rate, which is perhaps the most widely accepted mechanism for neural coding (Adrian and Zotterman, 1926; Shadlen and Newsome, 1994), could be such a mechanism. The presence of oscillations does not abolish the tuning of firing rates (Friedman-Hill et al., 2000; Liu and Newsome, 2006; Ardid et al., 2010). In fact, there is evidence that such tuning might even be enhanced in the presence of oscillations (Paik et al., 2009; Womelsdorf et al., 2012). Therefore, in the same volume of space, neurons coding for the currently relevant function would increase their firing rate, and because of phase-locking the coincidence of their spikes would amplify the signal they emit, while neurons coding for other functions would decrease their firing and would not avail themselves of this effect. Such a scheme makes further sense considering that most cortical neurons fire at low rates (Abeles et al., 1990; Shafi et al., 2007; Hromádka et al., 2008) and thus spurious coincidence firing will hence be small. Viewed in this way, oscillatory spatial computation is not an independent computational process but rather works in conjunction with previously found coding schemes such as rate coding, in order to enhance the temporal precision of functionally congruent populations.

\section{RELATIONSHIP WITH PHASE-OF-FIRING CODING}

Here we propose a model that does not require individual coordination of the timing of each neuron according to task demands. An earlier, more complex model, termed phase-offiring (PoF) coding assumes that information is present in the phase at spike firing of each neuron (Perkel and Bullock, 1968; Hopfield, 1995; Wang, 2010). Such coding has been found mostly at lower frequencies than gamma, for example, theta oscillations in the hippocampus (Huxter et al., 2008) or alpha oscillations in the auditory cortex (Kayser et al., 2009). One study described 
PoF coding in the primary visual cortex (Montemurro et al., 2008). They reported that such coding, at low frequencies of oscillations, carried up to 54\% more information about natural stimuli than spike counts alone. The information about the stimuli decreased for higher frequencies and was negligible for frequencies higher than $24 \mathrm{~Hz}$. However, there have also been descriptions of PoF coding for gamma-band frequencies in cortex. For example, Havenith et al. (2011) reported that different sequences of neurons fired in response to different stimuli, and that these sequences were related to $20-60 \mathrm{~Hz}$ oscillations. Other descriptions of gamma-band PoF coding have been reported as well (Masquelier et al., 2009). We proceed to examine the relationship between PoF coding and the proposal outlined here.

Clearly, PoF coding enables a higher information rate, as the information transmitted by the phase of each spike allows for a much richer representation with the same number of spikes. On the other hand, the single-phase spatial computation described here is more robust to noise since temporal jittering of spike timing would not lead to erroneous encoding. Rather, it implies less efficacious coincidences, which can be easily remedied by increasing the number of neurons involved, or the amplitude of oscillations (which is tightly related to the phase-locking and hence the temporal specificity of firing). For PoF coding, we expect that as the oscillation frequency increases, the detrimental effect of noise will increase as well, because as the oscillation cycle shortens each temporal jittering error reflects a larger phase error. Additional difficulties related to PoF coding at high frequencies have been considered as well (Montemurro et al., 2008; Wang, 2010). Thus, the two coding schemes can be seen as complementary: in low noise or low frequency regimes, PoF coding may provide powerful, high-throughput coding, while in the presence of increased noise or higher frequencies, singlephase spatial computation can provide a robust alternative. In this context is worth noting that it might be hard to disassociate between PoF and single-phase spatial-computation by standard experimental measures. For instance, a realistic example of spatial computation might not present exactly a zero-phase difference across all sites; the generation of the spatial distribution of oscillations may involve that oscillations in some sites start earlier than in others, resulting in small phase differences across sites. Since these differences depend on the spatial distribution of oscillations, they are stimulus dependent. Thus, repeatable, stimulus-dependent differences in phase will result in stimulusdependent differences in the timing of neuronal firing for neurons in different sites, which is commonly considered a characteristic of a PoF code. To differentiate between the two coding schemes we propose the following experiments and data analysis procedures: (1) recordings of spiking activity with high spatial resolution should be performed, after which a spatial analysis can be effectuated to determine whether the differences in timing of neuronal firing can be accounted for by the spatial location of the neurons. A strong relationship between spatial location and phase of firing would support the spatial computation hypothesis; (2) simultaneous recordings from multiple connected sites can be used to assess whether the differences in timing of firing (in the upstream neurons) have an effect on the firing patterns and coding capabilities of downstream neurons. If upstream neurons evidence multiple preferred firing phases which have a distinct effect on the downstream population, this can be construed as support for PoF coding.

\section{MECHANISM}

Although the mechanism of generation and maintenance of cortical gamma oscillations is still subject to intensive research, there is converging evidence that inhibitory subpopulations are critically involved in the formation of this oscillatory process (Buzsáki and Wang, 2012). Networks of inhibitory interneurons have been shown to mediate $\sim 40 \mathrm{~Hz}$ oscillations in vitro (Whittington et al., 1995); rhythmic activation of fast-spiking inhibitory neurons (but not regular-spiking excitatory neurons) is necessary for the generation of these oscillations in cortex in vivo (Cardin et al., 2009). One study reported a relationship between the ability of humans to discriminate between different orientations and the concentration of GABA, as well as the frequency of stimulus induced gamma oscillations in V1 (Edden et al., 2009). These results imply that studying the spatial connectivity patterns and spatial network properties of inhibitory subpopulations is an essential step in understanding the mechanism for generation and maintenance of specific spatial distributions of oscillations. The inhibitory network may play a key role in selecting between the different oscillatory spatial configurations according to task demands.

\section{LOCAL VS. DISTANT REMOTE SPATIAL COMPUTATION}

Gamma oscillations have frequently been described with regard to local processing (reviewed in Donner and Siegel, 2011). Spatial modulation of coincident firing is a plausible mechanism to support such processing. However, coherent gamma oscillations for spatially-remote regions have been described as well (Engel et al., 1991; Fries et al., 2001; Doesburg et al., 2008). Thus, computationally relevant spatial distribution patterns of oscillation could in principle involve spatially discontinuous topologies, even between hemispheres (Engel et al., 1991; König et al., 1995; Gregoriou et al., 2009).

\section{LIMITATIONS AND FUTURE PROSPECTS}

Most of the evidence we have described is the outcome of recordings from the neocortex. There may be characteristic differences in neuronal firing patterns in other brain areas where the oscillatory spatial computation described here is less likely. For example, in the hippocampus, pyramidal neurons typically have much weaker phase-locking to gamma oscillations, and population histograms of preferred phases are much broader, allowing for the separation of the pyramidal population into clusters based on their preferred firing phase (Senior et al., 2008). Thus, we expect a decrease in the precision of coincident firing under these circumstances, where the more prominent feature appears to be phase-coding with respect to theta oscillations (Buzsáki, 2002).

We have exclusively analyzed correlations arising from an oscillatory process in the gamma range, but in cortex there is evidence for both oscillatory and non-oscillatory modes of correlated activity (Eckhorn, 1994; Baker et al., 2001). The current framework does not account for non-oscillatory synchrony and accordingly does not aim to encompass the full gamut of possible modes of neural coordination but rather provide a simple 
explanation as to the role of oscillatory processes in the gamma range with regard to functional neuronal patterns.

Finally, it is clear that compared to the vast array of studies on gamma oscillations, there are few systematic spatial analyses of oscillatory properties. This state of affairs is likely to change as result of technological advances such as dense microelectrode arrays, high-resolution imaging and spatially-precise optogenetic manipulations. Future experiments using these methodologies for simultaneous recordings from identified neuronal populations will be better equipped to characterize the functional importance of the spatial properties of oscillations and single unit synchrony patterns.

\section{ACKNOWLEDGMENTS}

We thank Itai Novick for helpful discussions. This work was supported in part by the Binational Science Foundation (BSF), the Israel Science Foundation, special contributions from the Rosetrees Trust, the Gatsby Charitable Foundation, and the Ida Baruch fund. Ben Engelhard was partly supported by the Felix M. Katar fund and the Khazzam fund.

\section{REFERENCES}

Abeles, M. (1982). Local Cortical Circuits: An Electrophysiological Study. Berlin: Springer.

Abeles, M. (1991). Corticonics: Neural Circuits of the Cerebral Cortex. New York: Cambridge University Press.

Abeles, M., Vaadia, E., and Bergman, H. (1990). Firing patterns of single units in the prefrontal cortex and neural network models. Network 1, 13-25. doi: 10 . 1088/0954-898x/1/1/002

Adrian, E. D., and Zotterman, Y. (1926). The impulses produced by sensory nerveendings: Part II. The response of a Single End-Organ. J. Physiol. 61, 151-171.

Ardid, S., Wang, X. J., Gomez-Cabrero, D., and Compte, A. (2010). Reconciling coherent oscillation with modulation of irregular spiking activity in selective attention: gamma-range synchronization between sensory and executive cortical areas. J. Neurosci. 30, 2856-2870. doi: 10.1523/jneurosci.4222-09.2010

Azouz, R., and Gray, C. M. (2000). Dynamic spike threshold reveals a mechanism for synaptic coincidence detection in cortical neurons in vivo. Proc. Natl. Acad. Sci. U S A 97, 8110-8115. doi: 10.1073/pnas.130200797

Azouz, R., and Gray, C. M. (2003). Adaptive coincidence detection and dynamic gain control in visual cortical neurons in vivo. Neuron 37, 513-523. doi: 10. 1016/s0896-6273(02)01186-8

Baker, S. N., Spinks, R., Jackson, A., and Lemon, R. N. (2001). Synchronization in monkey motor cortex during a precision grip task. I. Task-dependent modulation in single-unit synchrony. J. Neurophysiol. 85, 869-885.

Bašar, E., and Güntekin, B. (2008). A review of brain oscillations in cognitive disorders and the role of neurotransmitters. Brain Res. 1235, 172-193. doi: 10. 1016/j.brainres.2008.06.103

Ben-Shaul, Y., Stark, E., Asher, I., Drori, R., Nadasdy, Z., and Abeles, M. (2003). Dynamical organization of directional tuning in the primate premotor and primary motor cortex. J. Neurophysiol. 89, 1136-1142. doi: 10.1152/jn.00364. 2002

Berger, H. (1929). über das Elektroenkephalogramm des menschen. Arch. Psychiatr. Nervenkr. 87, 527-570.

Brosch, M., Budinger, E., and Scheich, H. (2002). Stimulus-related gamma oscillations in primate auditory cortex. J. Neurophysiol. 87, 2715-2725.

Bruno, R. M., and Sakmann, B. (2006). Cortex is driven by weak but synchronously active thalamocortical synapses. Science 312, 1622-1627. doi: 10.1126/science. 1124593

Bruns, A., Eckhorn, R., Jokeit, H., and Ebner, A. (2000). Amplitude envelope correlation detects coupling among incoherent brain signals. Neuroreport 11 , 1509-1514. doi: 10.1097/00001756-200005150-00028

Burns, S. P., Xing, D., and Shapley, R. M. (2011). Is gamma-band activity in the local field potential of V1 cortex a "clock" or filtered noise? J. Neurosci. 31, 9658-9664. doi: 10.1523/jneurosci.0660-11.2011
Buzsáki, G. (2002). Theta oscillations in the hippocampus. Neuron 33, 325-340. doi: 10.1016/s0896-6273(02)00586-x

Buzsáki, G. (2006). Rhythms of the Brain. New York: Oxford University Press.

Buzsáki, G., and Draguhn, A. (2004). Neuronal oscillations in cortical networks. Science 304, 1926-1929. doi: 10.1126/science.1099745

Buzsáki, G., and Wang, X. J. (2012). Mechanisms of gamma oscillations. Annu. Rev. Neurosci. 35, 203-225. doi: 10.1146/annurev-neuro-062111-150444

Cardin, J. A., Carlén, M., Meletis, K., Knoblich, U., Zhang, F., Deisseroth, K., et al. (2009). Driving fast-spiking cells induces gamma rhythm and controls sensory responses. Nature 459, 663-667. doi: 10.1038/nature08002

Denker, M., Roux, S., Lindén, H., Diesmann, M., Riehle, A., and Grün, S. (2011). The local field potential reflects surplus spike synchrony. Cereb. Cortex 21, 26812695. doi: 10.1093/cercor/bhr040

Doesburg, S. M., Emberson, L. L., Rahi, A., Cameron, D., and Ward, L. M. (2008). Asynchrony from synchrony: long-range gamma-band neural synchrony accompanies perception of audiovisual speech asynchrony. Exp. Brain Res. 185, 11-20. doi: 10.1007/s00221-007-1127-5

Donner, T. H., and Siegel, M. (2011). A framework for local cortical oscillation patterns. Trends Cogn. Sci. 15, 191-199. doi: 10.1016/j.tics.2011.03.007

Eckhorn, R. (1994). Oscillatory and non-oscillatory synchronizations in the visual cortex and their possible roles in associations of visual features. Prog. Brain Res. 102, 405-426. doi: 10.1016/s0079-6123(08)60556-7

Edden, R. A., Muthukumaraswamy, S. D., Freeman, T. C., and Singh, K. D. (2009). Orientation discrimination performance is predicted by GABA concentration and gamma oscillation frequency in human primary visual cortex. J. Neurosci. 29, 15721-15726. doi: 10.1523/jneurosci.4426-09.2009

Engel, A., König, P., Kreiter, A., and Singer, W. (1991). Interhemispheric synchronization of oscillatory neuronal responses in cat visual cortex. Science 252, $1177-$ 1179. doi: 10.1126/science.252.5009.1177

Engelhard, B., Ozeri, N., Israel, Z., Bergman, H., and Vaadia, E. (2013). Inducing gamma oscillations and precise spike synchrony by operant conditioning via brain-machine interface. Neuron 77, 361-375. doi: 10.1016/j.neuron.2012. 11.015

Friedman-Hill, S., Maldonado, P. E., and Gray, C. M. (2000). Dynamics of striate cortical activity in the alert macaque: I. Incidence and stimulus-dependence of gamma-band neuronal oscillations. Cereb. Cortex 10, 1105-1116. doi: 10. 1093/cercor/10.11.1105

Fries, P. (2009). Neuronal gamma-band synchronization as a fundamental process in cortical computation. Annu. Rev. Neurosci. 32, 209-224. doi: 10. 1146/annurev.neuro.051508.135603

Fries, P., Neuenschwander, S., Engel, A. K., Goebel, R., and Singer, W. (2001). Rapid feature selective neuronal synchronization through correlated latency shifting. Nat. Neurosci. 4, 194-200. doi: 10.1126/science.1055465

Georgopoulos, A. P., Merchant, H., Naselaris, T., and Amirikian, B. (2007). Mapping of the preferred direction in the motor cortex. Proc. Natl. Acad. Sci. U S A 104, 11068-11072. doi: 10.1073/pnas.0611597104

Gray, C. M., and Singer, W. (1989). Stimulus-specific neuronal oscillations in orientation columns of cat visual cortex. Proc. Natl. Acad. Sci. U S A 86, 1698 1702. doi: $10.1073 /$ pnas.86.5.1698

Graziano, M. S., Taylor, C. S., and Moore, T. (2002). Complex movements evoked by microstimulation of precentral cortex. Neuron 34, 841-851. doi: 10 . 1016/s0896-6273(02)00698-0

Gregoriou, G. G., Gotts, S. J., Zhou, H., and Desimone, R. (2009). High-frequency, long-range coupling between prefrontal and visual cortex during attention. Science 324, 1207-1210. doi: 10.1126/science.1171402

Hasenstaub, A., Shu, Y., Haider, B., Kraushaar, U., Duque, A., and McCormick, D. A. (2005). Inhibitory postsynaptic potentials carry synchronized frequency information in active cortical networks. Neuron 47, 423-435. doi: 10.1016/j. neuron.2005.06.016

Havenith, M. N., Yu, S., Biederlack, J., Chen, N. H., Singer, W., and Nikolić, D. (2011). Synchrony makes neurons fire in sequence and stimulus properties determine who is ahead. J. Neurosci. 31, 8570-8584. doi: 10.1523/jneurosci. 2817-10.2011

Henrie, J. A., and Shapley, R. (2005). LFP power spectra in V1 cortex: the graded effect of stimulus contrast. J. Neurophysiol. 94, 479-490. doi: 10.1152/jn.00919. 2004

Herrmann, C. S., Fründ, I., and Lenz, D. (2010). Human gamma-band activity: a review on cognitive and behavioral correlates and network models. Neurosci. Biobehav. Rev. 34, 981-992. doi: 10.1016/j.neubiorev.2009.09.001 
Hopfield, J. J. (1995). Pattern recognition computation using action potential timing for stimulus representation. Nature 376, 33-36. doi: 10.1038/376 $033 \mathrm{a} 0$

Hromádka, T., Deweese, M. R., and Zador, A. M. (2008). Sparse representation of sounds in the unanesthetized auditory cortex. PLoS Biol. 6:e16. doi: 10. 1371/journal.pbio.0060016

Huxter, J. R., Senior, T. J., Allen, K., and Csicsvari, J. (2008). Theta phase-specific codes for two-dimensional position, trajectory and heading in the hippocampus. Nat. Neurosci. 11, 587-594. doi: 10.1038/nn.2106

Jacobs, J., and Kahana, M. J. (2009). Neural representations of individual stimuli in humans revealed by gamma-band electrocorticographic activity. J. Neurosci. 29, 10203-10214. doi: 10.1523/jneurosci.2187-09.2009

Jacobs, J., and Kahana, M. J. (2010). Direct brain recordings fuel advances in cognitive electrophysiology. Trends Cogn. Sci. 14, 162-171. doi: 10.1016/j.tics. 2010.01.005

Kayser, C., Montemurro, M. A., Logothetis, N. K., and Panzeri, S. (2009). Spikephase coding boosts and stabilizes information carried by spatial and temporal spike patterns. Neuron 61, 597-608. doi: 10.1016/j.neuron.2009.01.008

König, P., Engel, A. K., and Singer, W. (1995). Relation between oscillatory activity and long-range synchronization in cat visual cortex. Proc. Natl. Acad. Sci. U S A 92, 290-294. doi: 10.1073/pnas.92.1.290

König, P., Engel, A. K., and Singer, W. (1996). Integrator or coincidence detector? The role of the cortical neuron revisited. Trends Neurosci. 19, 130-137. doi: 10. 1016/s0166-2236(96)80019-1

Liu, J., and Newsome, W. T. (2006). Local field potential in cortical area MT: stimulus tuning and behavioral correlations. J. Neurosci. 26, 7779-7790. doi: 10. 1523/jneurosci.5052-05.2006

Logothetis, N. K., Pauls, J., Augath, M., Trinath, T., and Oeltermann, A. (2001). Neurophysiological investigation of the basis of the fMRI signal. Nature 412, 150-157. doi: $10.1038 / 35084005$

London, M., and Häusser, M. (2005). Dendritic computation. Annu. Rev. Neurosci. 28, 503-532. doi: 10.1146/annurev.neuro.28.061604.135703

Lutzenberger, W., Pulvermüller, F., Elbert, T., and Birbaumer, N. (1995). Visual stimulation alters local $40-\mathrm{Hz}$ responses in humans: an EEG-study. Neurosci. Lett. 183, 39-42. doi: 10.1016/0304-3940(94)11109-v

Macdonald, K. D., Fifkova, E., Jones, M. S., and Barth, D. S. (1998). Focal stimulation of the thalamic reticular nucleus induces focal gamma waves in cortex. J. Neurophysiol. 79, 474-477.

Masquelier, T., Hugues, E., Deco, G., and Thorpe, S. J. (2009). Oscillations, phaseof-firing coding and spike timing-dependent plasticity: an efficient learning scheme. J. Neurosci. 29, 13484-13493. doi: 10.1523/jneurosci.2207-09.2009

Mesulam, M. M. (1998). From sensation to cognition. Brain 121(Pt 6), 1013-1052. doi: 10.1093/brain/121.6.1013

Montemurro, M. A., Rasch, M. J., Murayama, Y., Logothetis, N. K., and Panzeri, S. (2008). Phase-of-firing coding of natural visual stimuli in primary visual cortex. Curr. Biol. 18, 375-380. doi: 10.1016/j.cub.2008.02.023

Murthy, V. N., and Fetz, E. E. (1996). Synchronization of neurons during local field potential oscillations in sensorimotor cortex of awake monkeys. J. Neurophysiol. 76, 3968-3982.

Paik, S. B., Kumar, T., and Glaser, D. A. (2009). Spontaneous local gamma oscillation selectively enhances neural network responsiveness. PLoS Comput. Biol. 5:e1000342. doi: 10.1371/journal.pcbi.1000342

Palanca, B. J., and DeAngelis, G. C. (2005). Does neuronal synchrony underlie visual feature grouping? Neuron 46, 333-346. doi: 10.1016/j.neuron.2005. 03.002

Penfield, W., and Boldrey, E. (1937). Somatic motor and sensory representation in the cerebral cortex of man as studied by electrical stimulation. Brain 60 , 389-443. doi: 10.1093/brain/60.4.389

Perkel, D. H., and Bullock, T. H. (1968). Neural coding. Neurosci. Res. Prog. Sum. 3, 405-527.

Rathelot, J. A., and Strick, P. L. (2006). Muscle representation in the macaque motor cortex: an anatomical perspective. Proc. Natl. Acad. Sci. U S A 103, 8257-8262. doi: 10.1073/pnas.0602933103

Ringach, D. L., Shapley, R. M., and Hawken, M. J. (2002). Orientation selectivity in macaque V1: diversity and laminar dependence. J. Neurosci. 22, 5639-5651.

Rols, G., Tallon-Baudry, C., Girard, P., Bertrand, O., and Bullier, J. (2001). Cortical mapping of gamma oscillations in areas V1 and V4 of the macaque monkey. Vis. Neurosci. 18, 527-540. doi: 10.1017/s0952523801184038
Rothschild, G., Nelken, I., and Mizrahi, A. (2010). Functional organization and population dynamics in the mouse primary auditory cortex. Nat. Neurosci. 13, 353-360. doi: 10.1038/nn.2484

Scherberger, H., Jarvis, M. R., and Andersen, R. A. (2005). Cortical local field potential encodes movement intentions in the posterior parietal cortex. Neuron 46, 347-354. doi: 10.1016/j.neuron.2005.03.004

Schieber, M. H., and Hibbard, L. S. (1993). How somatotopic is the motor cortex hand area? Science 261, 489-492. doi: 10.1126/science.8332915

Schnitzler, A., and Gross, J. (2005). Normal and pathological oscillatory communication in the brain. Nat. Rev. Neurosci. 6, 285-296. doi: 10.1038/nrn 1650

Senior, T. J., Huxter, J. R., Allen, K., O'Neill, J., and Csicsvari, J. (2008). Gamma oscillatory firing reveals distinct populations of pyramidal cells in the CA1 region of the hippocampus. J. Neurosci. 28, 2274-2286. doi: 10. 1523/JNEUROSCI.4669-07.2008

Shadlen, M. N., and Newsome, W. T. (1994). Noise, neural codes and cortical organization. Curr. Opin. Neurobiol. 4, 569-579. doi: 10.1016/0959-4388(94) 90059-0

Shafi, M., Zhou, Y., Quintana, J., Chow, C., Fuster, J., and Bodner, M. (2007). Variability in neuronal activity in primate cortex during working memory tasks. Neuroscience 146, 1082-1108. doi: 10.1016/j.neuroscience.2006.12.072

Siegel, M., Donner, T. H., and Engel, A. K. (2012). Spectral fingerprints of large-scale neuronal interactions. Nat. Rev. Neurosci. 13, 121-134. doi: 10. 1038/nrn3137

Singer, W., and Gray, C. M. (1995). Visual feature integration and the temporal correlation hypothesis. Annu. Rev. Neurosci. 18, 555-586. doi: 10.1146/annurev. neuro.18.1.555

Tallon-Baudry, C., Bertrand, O., Peronnet, F., and Pernier, J. (1998). Induced gamma-band activity during the delay of a visual short-term memory task in humans. J. Neurosci. 18, 4244-4254.

Taylor, K., Mandon, S., Freiwald, W. A., and Kreiter, A. K. (2005). Coherent oscillatory activity in monkey area $\mathrm{v} 4$ predicts successful allocation of attention. Cereb. Cortex 15, 1424-1437. doi: 10.1093/cercor/bhi023

Uhlhaas, P. J., and Singer, W. (2006). Neural synchrony in brain disorders: relevance for cognitive dysfunctions and pathophysiology. Neuron 52, 155-168. doi: 10. 1016/j.neuron.2006.09.020

Uhlhaas, P. J., and Singer, W. (2012). Neuronal dynamics and neuropsychiatric disorders: toward a translational paradigm for dysfunctional large-scale networks. Neuron 75, 963-980. doi: 10.1016/j.neuron.2012.09.004

Wang, X. J. (2010). Neurophysiological and computational principles of cortical rhythms in cognition. Physiol. Rev. 90, 1195-1268. doi: 10.1152/physrev.00035. 2008

Whittington, M. A., Traub, R. D., and Jefferys, J. G. (1995). Synchronized oscillations in interneuron networks driven by metabotropic glutamate receptor activation. Nature 373, 612-615. doi: 10.1038/373612a0

Womelsdorf, T., Lima, B., Vinck, M., Oostenveld, R., Singer, W., Neuenschwander, S., et al. (2012). Orientation selectivity and noise correlation in awake monkey area V1 are modulated by the $\gamma$ cycle. Proc. Natl. Acad. Sci. U S A 109, 43024307. doi: $10.1073 /$ pnas. 1114223109

Zhang, Z. G., Hu, L., Hung, Y. S., Mouraux, A., and Iannetti, G. D. (2012). Gamma-band oscillations in the primary somatosensory cortex-a direct and obligatory correlate of subjective pain intensity. J. Neurosci. 32, 7429-7438. doi: 10.1523/jneurosci.5877-11.2012

Conflict of Interest Statement: The authors declare that the research was conducted in the absence of any commercial or financial relationships that could be construed as a potential conflict of interest.

Received: 31 January 2013; accepted: 25 August 2014; published online: 09 September 2014.

Citation: Engelhard B and Vaadia E (2014) Spatial computation with gamma oscillations. Front. Syst. Neurosci. 8:165. doi: 10.3389/fnsys.2014.00165

This article was submitted to the journal Frontiers in Systems Neuroscience.

Copyright $\odot 2014$ Engelhard and Vaadia. This is an open-access article distributed under the terms of the Creative Commons Attribution License (CC BY). The use, distribution or reproduction in other forums is permitted, provided the original author(s) or licensor are credited and that the original publication in this journal is cited, in accordance with accepted academic practice. No use, distribution or reproduction is permitted which does not comply with these terms. 